\title{
Potential Therapeutics: Toe Hold in the Fight against Zika Virus
}

\section{Azizul Haque ${ }^{1 * \#}$ and Anudeep B Pant ${ }^{2 *}$}

${ }^{1}$ Geisel School of Medicine at Dartmouth, Lebanon, NH, USA

${ }^{2}$ School of Public Health and Tropical Medicine at Tulane University, New Orleans, LA, USA

\#This manuscript is dedicated to the memory of Dr. Sakhina Begum-Haque, who has been a constant inspiration for both the authors to pursue science for the good of human health

\begin{abstract}
No specific therapeutic agent or vaccine has yet been approved for the treatment or prevention of Zika virus infection, which may induce microcephaly in babies born to infected mothers and Guillain-Barré Syndrome in adults. Although the reported numbers of Zika cases have fallen in the last few months, multiple outbreaks of Zika virus and the likelihood of future exposure highlights the need for better preparedness and the development of effective treatments. Here, we briefly review both existing and future options for anti-Zika therapy by taking into account the epitome of Zika virus neurotropism. We project the mechanistic hypotheses of several potential drugs under development and propose the repurposing of drugs with known properties that are medically approved. Furthermore, we underline the importance of combinational therapy for better treatment outcomes for Zika patients. Our paper also discusses strategies for identifying potential target populations to develop therapeutics and test their anti-Zika properties.
\end{abstract}

Keywords: Zika; Virus; Therapeutic; Repurposing; Antibiotic drug

Abbreviations: ZIKV: Zika Virus; GBS: Guillain-Barré Syndrome; DENV: Dengue Virus; CHIKV: Chikungunya Virus; hNPCs: Human Cortical Neural Progenitor Cells; NS: Non-structural

\section{Introduction}

The Zika Virus (ZIKV) was discovered in monkeys in 1947 in Uganda's Zika Forest [1,2], and the first human case was recorded in Nigeria in 1954 [3]. For decades it did not appear to pose much of a threat to humans. Following a large-scale outbreak in the Micronesian island of Yap in 2007, researchers began to take an interest in the Zika virus [4].

Since 2014, the virus has swept through the Caribbean and Latin America infecting millions of people [5]. Zika cases have also been detected in the continental USA, particularly in the state of Florida. As of May 26, 2017, the outbreak has affected 5,300 individuals in the US, with an additional 36,582 cases reported in US territories [6]. ZIKV poses a threat to uninfected populations in other parts of the world due to the widespread presence of the insect vector [7]. Further, accidental importation by infected individuals traveling from regions where the virus is endemic remains a possibility. Zika virus is mainly transmitted by Aedes aegypti mosquitoes [8,9], however, the risk of transmission is compounded by the virus' capability of being transmitted via sexual intercourse [10] and blood transfusion [11] due to its persistence in semen [12] and blood respectively. Furthermore, infective ZIKV particles have been detected in the saliva, urine, and breast milk of infected patients [13-15]. Although infection rates have diminished, reports of emerging cases each week clearly demonstrate that the virus is far from eradicated. Due to the impact of the upcoming El Niño [16], in conjunction with the return of summer months facilitating the breeding of mosquitoes and increased summer travel to Zika endemic areas, more cases of infection are likely to resurface. We postulate that like dengue virus, a closely related flavivirus, Zika is likely to be around for the foreseeable future.

This review summarizes and evaluates the potential of current experimental candidate drugs for treating Zika virus disease and associated neurological disorders with regard to their feasibility and use in the clinic. In the absence of FDA approved drugs, the repurposing of existing drugs with established safety records and combination therapy may contribute to the amelioration of Zika virus pathogenicity. These points have been highlighted in this paper.

\section{Zika Virus and Disease}

The clinical presentation of ZIKV infection is not specific and can be confused with other diseases, especially dengue (DENV) and chikungunya (CHIKV). Most clinical cases presented with mild symptoms characterized by low-grade fever, maculopapular rash, arthralgia, and conjunctivitis. Prior to the French Polynesian epidemic, during which severe neurological complications (GBS) were confirmed, ZIKV was believed to cause only mild disease. The world became more aware of the virus in 2015, with reports of hundreds of cases of microcephaly in Brazilian new-borns whose mothers reported symptoms related to Zika viral infection during pregnancy.

ZIKV infection during pregnancy may be linked to fetal neurological complications that include brain damage and microcephaly $[5,17,18]$. It remains unknown how ZIKV can gain access to the fetal brain and how the viral infection relates to fetal brain malformations. Tang et al. report that ZIKV directly infects human cortical neural progenitor cells (hNPCs) with high efficiency, resulting in transcriptional dysregulation and stunted growth of this cell population. The finding raises critical questions about the pathological effects on neurons and other neural cell types in the brain, as well as potential long-term consequences (Figure 1).

Studies have shown that the candidate viral entry receptor, AXL, is highly expressed by human radial glial cells, astrocytes, endothelial cells, and microglia in the developing human cortex and by progenitor

*Corresponding author: Azizul Haque, Geisel School of Medicine at Dartmouth Lebanon, NH, USA, Tel: 603653 9946; E-mail: azizul.haque@dartmouth.edu

Received June 15, 2017; Accepted June 26, 2017; Published July 04, 2017

Citation: Haque A, Pant AB (2017) Potential Therapeutics: To Hold in the Fight against Zika Virus. J Bioanal Biomed 9: 177-185. doi:10.4172/1948-593X.1000175

Copyright: $\odot 2017$ Haque A, et al. This is an open-access article distributed under the terms of the Creative Commons Attribution License, which permits unrestricted use, distribution, and reproduction in any medium, provided the original author and source are credited. 


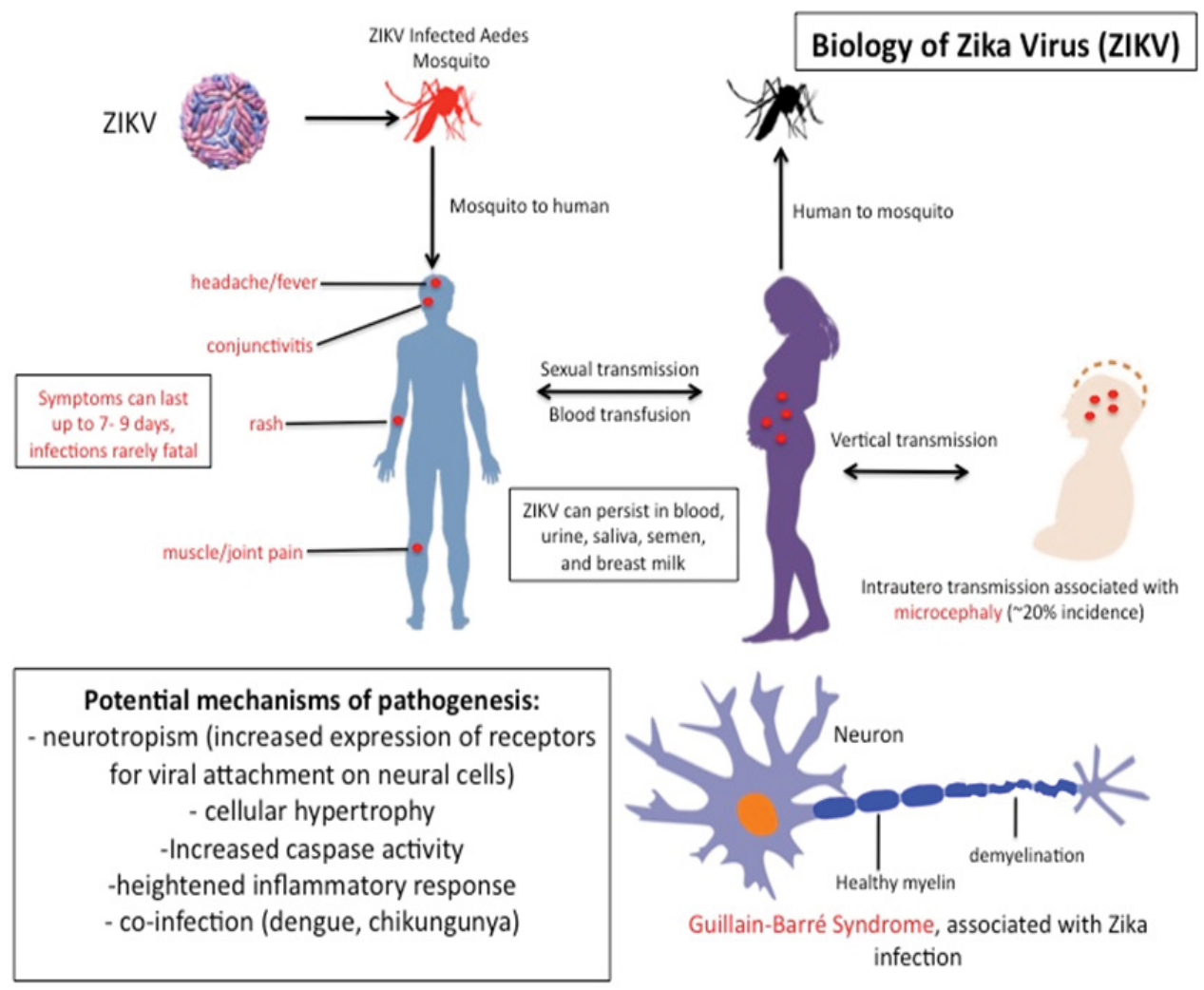

Figure 1: Biology of Zika Virus (ZIKV). This figure shows modes of transmission, illustrates disease symptoms and two critical pathological manifestations (microcephaly and Guillain-Barré Syndrome (GBS)) associated with Zika infection. Potential mechanisms of pathogenesis have also been highlighted.

cells in developing retina [19]. Nowakowski and colleagues survey the expression of candidate ZIKV entry proteins to suggest that high AXL expression in neural stem cells may render this population selectively vulnerable to viral infection [19]. Studies have demonstrated that blocking or silencing AXL reduces infectivity in cultured fibroblasts and alveolar epithelial cells by as much as 90\% [20]. Future studies will be needed to test the hypothesis whether AXL expression alone determines the cellular tropism for ZIKV in the developing human brain or if other binding factors, including genes expressed at low levels, may be involved.

Microcephaly cases in Brazil started to rise around 6 months after authorities confirmed Zika transmission there, suggesting that the defect might have been caused by in utero exposure to the virus $[17,21]$. In addition, researchers in Brazil have found traces of the virus or antibodies to it, in the amniotic fluid, brains, or spinal fluid of 15 fetuses and babies diagnosed with microcephaly $[5,17,22]$. This is suggestive, but not conclusive as not all babies born to Zika infected mothers will develop microcephaly. It is plausible that specific prototypes of Zika virus, while in high titer, can induce microcephaly alone under certain pregnancy conditions. The titer of ZIKV in infected humans is currently unknown. In addition, many questions remain unanswered, including the frequency of transmission from mother to fetus, the frequency of adverse outcomes in fetal Zika virus infection, and the spectrum of pregnancy and neonatal outcomes associated with maternal Zika virus infection.

Some co-factors including co-infections with other microorganisms may also have a role in the development of microcephaly. Further studies are needed to unveil whether co-infection and subsequent infection by different arboviruses can affect the course of the disease, the occurrence of severe cases, and the modes of transmission (vertical, perinatal, sexual) $[10,23,24]$. Due to limited ZIKV diagnostic capacity and the over-lapping clinical features of ZIKV, DENV, and CHIKV, which also circulate in the Pacific, understanding the true extent of chikungunya-dengue co-infection remains a challenge.

Studies suggest that the virus may be toxic only while a fetus' brain is still developing its major structures during the first two months of pregnancy. Alternatively, ZIKV may persist in the body for a long period, which would explain why the virus is seen in stillborn babies with microcephaly. If the insult happened early on, then why is the virus present at seven months when the miscarriage occurs? It is likely that a combination of factors contribute to the pregnancy outcomes of Zika infected mothers and their babies. Another conundrum is pinpointing what factors makes certain women and babies so vulnerable since the vast majority of women infected with Zika go on to have healthy babies. Zika virus can infect pregnant women in all three trimesters [25], which suggests that maternal-fetal transmission of ZIKV can occur. Studies are needed to determine:

1. How the virus crosses the placenta to infect the fetal brain thus causing generalized growth restriction [25].

2. Whether the virus infects adult human brains, as ZIKV has recently been detected in the cerebrospinal fluid of adults [26,27]. Finally, other flaviviruses that use similar entry receptors have not been strongly associated with fetal brain abnormalities, and future work must examine potential changes in recent strains of ZIKV. 
Previous studies highlight a vast viral versus human peptide commonality. A search for ZIKV peptide occurrences in human proteins linked to Guillain-Barré-like syndromes also show a high, unexpected level of peptide sharing [28]. While the highly unusual GBS cluster is very suspicious, it does not confirm ZIKV as the antigenic stimulus leading to this autoimmune disease. Of note, further analyses using the Immune Epitope Data Base (IEDB) resource show that many of the shared human/viral peptides are endowed with immunological potential. The data indicate that immune reactions following ZIKV infection may provide a considerable source of cross-reactions with brain-specific proteins, and might contribute to the ZIKV-associated neuropathologic sequelae [28].

\section{Therapeutic Candidates to Treat ZIKV Patients}

Critical understanding of the structure, tropism, and pathogenesis of ZIKV is of paramount importance to develop effective therapeutics against ZIKV. Within a short time period, useful mouse and nonhuman primate disease models have been established, and pre-clinical evaluation of therapeutics has begun. A neuroprotective drug to treat/ cure Zika infection is urgently needed. In this paper we will review the existing and future options for anti-Zika therapy based on clinical findings and experimental studies.

\section{Aravive-S6}

It has recently been reported that ZIKV entry into cells requires the AXL ligand, Gas6, which bridges the viral particles to glial cells, where the virus is then internalized [29,30]. During the viral entry of the cell, the ZIKV/Gas6 complex activates AXL kinase activity, which dampens the interferon response to the virus and facilitates infection [29,31,32]. It has recently been reported that Aravive Biologic's engineered decoy AXL receptor, Aravive-S6, can block ZIKV infection by intercepting Gas6 to prevent AXL signaling [29]. These findings suggest a potential antiviral role for Aravive-S6 in addition to its anticancer activity.

\section{P300/CBP}

ZIKV antagonizes the host type I interferon (IFN-I) response by preventing JAK-STAT signaling, suggesting that suppression of this pathway is an important determinant of infection [33,34]. ZIKV NS5 expression resulted in proteasomal degradation of the IFN-regulated transcriptional activator STAT2 from humans, but not mice, which may explain the requirement for IFN deficiency to observe ZIKVinduced disease in mice [34]. Transcription adaptor P300/CBP (EP300/ CREBBP) has been shown to interact specifically with STAT2 protein, which is thought to be involved in the process of blocking IFN- $\alpha$ response by Zika. Dysregulation of the transcriptional and epigenetic functions of $\mathrm{CBP} / \mathrm{p} 300$ could be associated with Zika disease, thus making it a potential anti-Zika drug target (Table 1 and Figure 2).

\section{Repurposing Drugs for Treatment of Zika Patients}

The merit and scientific rationale for using existing therapies in the absence of FDA approved drugs, especially when confronting remerging and rapidly spreading viral infections, has previously been discussed [35]. These compounds could be directed at reducing or preventing Zika pathogenicity. In light of the current Zika epidemic, three potential treatments have been identified by screening 6,000 different compounds. These three drugs may offer pregnant women and their developing fetuses' protection against the damaging effects of Zika virus.

\section{Niclosamide}

Niclosamide appears to have antiviral properties that inhibit Zika from replicating. This drug is already on the market as a treatment for tapeworm, and it is well tolerated [36,37]. The mechanism by which this drug acts against Zika virus is poorly understood. It is known to inhibit several viruses in culture systems, including the Japanese encephalitis flavivirus does not need to be capitalized (JEV) [38,39]. Niclosamide's broad antiviral activity has been attributed to its ability to neutralize endolysosomal $\mathrm{pH}$ and interfere with $\mathrm{pH}$-dependent membrane fusion [40] which is an essential step in the common virus entry pathway. It seems that inhibition by niclosamide occurs at a post-entry step, such as replication $[40,41]$. Niclosamide is a category B drug, which indicates that no risk to fetuses has been found in animal studies due to its low toxicity in mammals [42]. The WHO recommends that niclosamide may be used during pregnancy because it has not been shown to be mutagenic, teratogenic or embryotoxic [43].

\begin{tabular}{|c|c|c|c|c|}
\hline Drug Name & Target & Test Results & Observations & Citation \\
\hline Aravive-s6 & Blocks AXL signaling & $\begin{array}{l}\text { Intercepts ZIKV/Gas6 complex to block AXL } \\
\text { signaling, thus inhibiting ZIKV infection }\end{array}$ & $\begin{array}{l}\text { Inhibition of AXL protects microglia and astrocytes from } \\
\text { ZIKV infection, reducing CNS viral load. }\end{array}$ & {$[29]$} \\
\hline P300/CBP & STAT2 protein & $\begin{array}{l}\text { P300/CBP and STAT2 interaction blocks IFN- } \alpha \\
\text { responses }\end{array}$ & $\begin{array}{l}\text { Dysregulation of P300/CBP could be associated with } \\
\text { ZIKV disease }\end{array}$ & {$[34]$} \\
\hline Niclosamide & $\begin{array}{l}\text { Neutralizes endosomal } \mathrm{pH} \\
\text { disrupting } \mathrm{pH} \text { dependent } \\
\text { membrane fusion }\end{array}$ & Inhibits other flaviviruses (JEV) in vitro & $\begin{array}{l}\text { Mechanism of action is poorly understood, inhibition } \\
\text { occurs post entry, most replication }\end{array}$ & {$[40]$} \\
\hline Emricasan & $\begin{array}{l}\text { Pan-caspase inhibitor } \\
\text { attenuates ZIKV induced } \\
\text { increases in caspase-3 activity }\end{array}$ & $\begin{array}{l}\text { Protects against } 3 \text { strains of ZIKV, protects human } \\
\text { neural progenitors in in vitro cultures }\end{array}$ & $\begin{array}{l}\text { Does not directly affect ZIKV, but may protect fetal brain } \\
\text { against damage }\end{array}$ & {$[41]$} \\
\hline $\begin{array}{l}\text { PHA- } \\
690509\end{array}$ & $\begin{array}{l}\text { Cyclin dependent kinase } \\
\text { inhibitor }\end{array}$ & $\begin{array}{l}\text { Inhibited replication of } 3 \text { strains of ZIKV in a dose } \\
\text { dependent manner }\end{array}$ & $\begin{array}{l}\text { Inhibits ZIKV infection at post-likely at viral RNA entry } \\
\text { stage, most replication }\end{array}$ & {$[41]$} \\
\hline Chloroquine & $\begin{array}{l}\text { Imported into acidic vesicles, } \\
\text { thus increasing } \mathrm{pH}\end{array}$ & $\begin{array}{l}\text { ZIKV pass through endosomes and require } \\
\text { acidification to exit }\end{array}$ & $\begin{array}{l}\text { Viral particles are trapped in endosomes, most } \\
\text { efficacious at inhibiting during fusion of the envelope } \\
\text { protein to the endosome membrane }\end{array}$ & {$[52]$} \\
\hline FTY720 & $\begin{array}{l}\text { S1P receptor modulator, } \\
\text { sequesters lymphocytes within } \\
\text { lymph nodes }\end{array}$ & $\begin{array}{l}\text { ZIKV infections have been associated with } \\
\text { increased inflammatory molecules, FTY20 has } \\
\text { been shown to limit inflammatory responses to } \\
\text { other viruses }\end{array}$ & $\begin{array}{l}\text { Immunomodulatory therapeutic would expand regulatory } \\
\text { cells secreting anti-inflammatory cytokines and could } \\
\text { dampen inflammatory ZIKV induced responses }\end{array}$ & {$[59]$} \\
\hline Rapamycin & $\begin{array}{l}\text { TOR cellular stress-response } \\
\text { pathway }\end{array}$ & $\begin{array}{l}\text { ZIKV induces cellular hypertrophy and growth } \\
\text { restriction through the TOR pathway }\end{array}$ & $\begin{array}{l}\text { Mice treated with rapamycin show increased resistance } \\
\text { to flu infection and may ameliorate ZIKV infection }\end{array}$ & {$[62]$} \\
\hline
\end{tabular}

Table 1: Anti-Zika therapies targeting host factors. This table shows repurposed and experimental drugs that target host factors and which may be efficacious in the treatment of ZIKV infection. Most of these drugs have been approved by the FDA and are widely available for treatment of Zika affected patients. 


\section{Targets for Potential Anti-Zika Drugs}

Fig 2A. Viral Targets

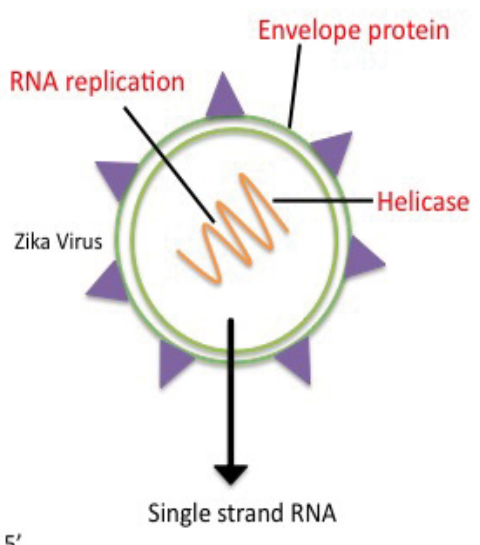

$5^{\prime}$

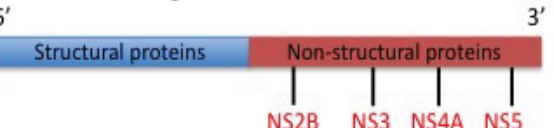

Fig 2B. Host Targets

AXL receptors on neural cells (radial glia, astrocytes, endothelial cells, microglia)
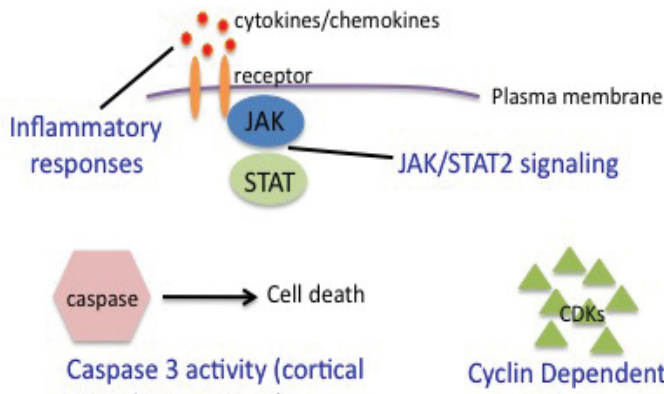
neural progenitors)

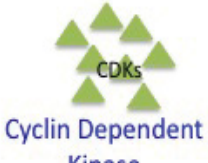

Kinase

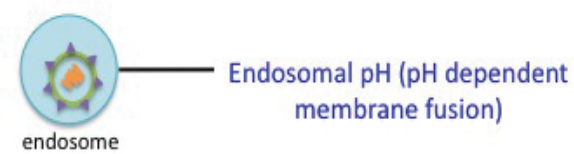

Figure 2: Targets for potential anti-Zika drugs. Panel (A) depicts possible viral targets for anti-Zika drug that include envelope (E) protein, non-structural proteins (NS2B, NS3, NS4A and NS5), helicase and RNA replication. Panel (B) shows potential host-targets that comprise AXL receptors on neural cells, elevated inflammatory responses (proinflammatory cytokines and JAK/STAT2 signaling), caspase 3 activity, cyclin dependent kinase, and endosomal pH affecting membrane fusion.

\section{Emricasan}

Investigators have identified a third medication awaiting U.S. FDA approval that doesn't directly act against Zika, but may be able to protect the brain cells of developing fetuses against damage. A pancaspase inhibitor, emricasan, inhibited ZIKV-induced increases in caspase-3 activity [44] and protected human cortical neural progenitors in both monolayer and three-dimensional organoid cultures [41]. Emricasan was identified as the most potent anti-cell-death compound, and provided protection against three ZIKV strains: MR766 (1947 Ugandan strain), FSS13025 (2010 Cambodian strain) and PRVABC59 (2015 Puerto Rican strain) [41]. This compound wouldn't necessarily be good for treating infections by itself because it can't stop the infection, however, it may be used it to buy time and protect the cells against infection.

\section{PHA-690509}

Another antiviral drug potentially effective against Zika is PHA690509. This is a medication currently in development, which works by interfering with gene expression [45]. PHA-690509, functions as a Cyclin-dependent kinase inhibitor (CDKi). This compound inhibited replication of all three strains of ZIKV, as measured by NS1 expression, in a dose-dependent manner [41]. PHA-690509 inhibits ZIKV infection at a post-entry stage, probably at the viral RNA replication step [41]. In vitro analyses demonstrate that treatment with PHA-690509 partially rescued the ZIKV-induced reduction of hNPC proliferation [41]. Furthermore, treatment in the absence of ZIKV infection showed a minimal effect on hNPC proliferation in brain organoid cultures, which model early human brain development in vitro.

While these three drugs have shown promise following several in vitro studies, much work needs to be done before they are available to humans. For example, researchers have to test in mice and primates whether the drugs protect against ZIKV infection in vivo, and whether they are safe to take during pregnancy.

\section{Chloroquine}

Chloroquine has been used as an antimalarial drug, antiinflammatory agent, and has shown antiviral activity against several viruses. Chloroquine, a 4 -aminoquinoline, is a weak base that is rapidly imported into acidic vesicles, increasing their $\mathrm{pH}[46,47]$. It is approved by the FDA to treat malaria and has long been prescribed prophylactically to pregnant women that are at risk of exposure to Plasmodium parasites $[48,49]$. Both chloroquine and the related compound, hydroxychloroquine, are used in the treatment of systemic lupus erythematous and rheumatoid arthritis due to their anti-inflammatory properties [50]. These antimalarial agents accumulate in endosomes and prevent acidification maturation, which is crucial in the activation of not only lysosomal enzymes but also innate immune receptors TLR7 and TLR9. ZIKV pass through endosomes; since the subsequent acidification is essential for exiting the endosomes [51], chloroquine and hydroxychloroquine could trap viral particles thus preventing their escape. Studies demonstrate a strong reduction in the release of ZIKV particles when the drug was added at $0 \mathrm{~h}$ post-infection, suggesting a higher impact on early stages of infection, possibly during fusion of the 
envelope protein to the endosome membrane [52]. Furthermore, it has recently been shown that chloroquine exhibits antiviral activity against ZIKV in Vero cells, human brain microvascular endothelial cells, and human neural stem cells [52]. Chloroquine has been shown to reduce the number of ZIKV-infected cells in vitro, and inhibit virus production and cell death promoted by ZIKV infection without cytotoxic effects. In addition, chloroquine treatment partially reverses morphological changes induced by ZIKV infection in mouse neurospheres [52].

\section{FTY720}

FTY720, also known as Fingolimod, is an FDA approved drug used to treat multiple sclerosis (MS) [53,54]. This is an oral S1P (sphingosine-1-phosphate) receptor modulator, which sequesters lymphocytes in lymph nodes, preventing them from contributing to an autoimmune reaction [55]. FTY720 has been shown to be a useful agent for prevention of transplant rejection and autoimmune diseases [56]. Studies analyzing the amniotic fluid of ZIKV-positive pregnant women with neonatal microcephaly show evidence of heightened immune activation, which manifested by the generation of several inflammatory cytokines/chemokines [57,58]. These results suggest that the immune activation caused by ZIKV infection in the uterine environment could also interfere with fetal development. Treatment with an immunomodulatory therapeutic that would promote the expansion of regulatory $\mathrm{T}$ and/or B cells secreting anti-inflammatory cytokines (IL-10, TGF- $ß$ ) could dampen immunopathologic responses in Zika patients. Recently, therapy with AAL-R, a chiral sphingosine analog of the clinical drug FTY720, was shown to limit inflammation and mortality in mice infected with a highly pathogenic $\mathrm{H} 1 \mathrm{~N} 1$ influenza virus [59]. Interestingly, this treatment did not alter the generation of influenza virus-neutralizing antibodies. Treatment of ZIKV infection with FTY720 in patients experiencing autoimmune complications could provide beneficial clinical outcomes.

\section{Targeting TOR Stress-response Pathway}

In a recent study using a fission yeast cell system, it has been shown that all ZIKV structural proteins, with the exception of Pr and two non-structural proteins (NS2B and NS4A), conferred cytopathic effects that included inhibition of growth/ proliferation, cell hypertrophy, cellcycle dysregulation, and cell death [60]. Genetic studies suggested that cellular hypertrophy and growth restriction were mediated through the Tor1 and Tip41 proteins of the TOR cellular stress-response pathway [60].

Rapamycin has been known to impact the TOR cellular pathway $[61,62]$ and could be exploited as an anti-Zika therapeutic. Rapamycin is used to help prevent organ rejection in transplant patients, which raises fears that it may dangerously suppress the immune system. A 2014 study conducted by Novartis, however, suggested otherwise; a rapamycin analogue called everolimus improved the immune response to an influenza vaccine in older people. In addition, mice treated with everolimus exhibited greater resistance against flu infection [63]. It would be valuable to investigate the outcomes of rapamycin and everolimus treatment for ZIKV infections in experimental models before conducting testing in humans.

\section{Antiviral Compounds in Zika Infection}

\section{Anti-influenza compounds against ZIKV}

Development of broad-spectrum cell-directed antivirals could be a valuable approach for the treatment of Zika. Researchers from the University of Helsinki have recently shown that three drugs, called obatoclax, saliphenylhalamide, and gemcitabine prevented synthesis of viral building blocks and production of new viruses at concentrations that were not toxic to cells [64]. In this study, human retinal pigment epithelial cells were infected with a Zika virus strain isolated from fetal brains. It was demonstrated that antivirals, particularly those that block influenza virus by targeting host cell factors, were also able to inhibit Zika virus infection. These antiviral drugs and their combinations are potent inhibitors of Zika virus-host cell interaction.

\section{Boceprevir, telaprevir and sofosbuvir}

In 2011, the US Food and Drug Administration approved two new antivirals, boceprevir and telaprevir, for treating hepatitis $\mathrm{C}$ virus (HCV) infection [65-67]. The drugs inhibit an important viral protein, the NS3-4A protease of HCV [68]. Non-structural protein 3 (NS3) is a protease and helicase, which is common to the Flavivirus genus $[69,70]$. On the basis of its similarity to the homologue encoded by the HCV, the flavivirus NS3 might be a promising drug target in regards to ZIKV. In addition, sofosbuvir, another nucleotide analog approved for the treatment of HCV, has shown potent inhibition of ZIKV infection in a murine model and in human neuronal stem cells [71].

Recently, a high-resolution 3D structure of ZIKV helicase has been presented [72]. Superposition of ZIKV helicase to Dengue virus type 4 helicase bound with a 12-mer ssRNA generated an important atomic model to analyze the pattern of nucleic acid binding, which provided structural insight into the RNA unwinding activity of ZIKV helicase [72]. The structure has revealed critical substrate-binding pockets for rational antiviral drug design. Pharmaceutical development of inhibitors targeting the RNA binding tunnel and the pivotal regulatory regions would be a plausible strategy for innovative anti-ZIKV therapies.

Further investigations on the effect of treatment with RNA aptamers, small peptides, antibodies and small molecules that specifically inhibit the flavivirus helicase may be exploited for improved treatment outcomes for Zika patients. Now may also be the time to initiate broader screens for small molecule Zika helicase inhibitors. Recent advances in understanding the molecular basis for helicase action might also spur interest in rationally designing compounds that might target key motifs or clefts. However, more sensitive, high-throughput Zika helicase assays need to be developed.

\section{Potent activity of BCX4430 against ZIKV}

BCX4430, a novel synthetic adenosine analogue, inhibits infection of distinct filoviruses in human cells [73]. Several assays indicate that BCX4430, a nucleoside RNA polymerase inhibitor, is metabolized to the active triphosphate (nucleotide) form by cellular kinases [73]. Recently, this compound has been tested against the African and Asian lineage ZIKV in cytopathic effect inhibition and virus yield reduction assays [74]. A mouse model of severe ZIKV infection, which recapitulates various human disease manifestations including peripheral virus replication, conjunctivitis, encephalitis and myelitis, was utilized to evaluate the efficacy of treatment [74]. Treatment of ZIKV-infected mice with BCX4430 significantly improved disease outcome even when treatment was initiated during the peak of viremia [74]. The drug nucleotide binds to the viral enzyme active site and becomes incorporated into the growing viral RNA strand, leading to premature chain termination [73]. It does not incorporate into human RNA or DNA [73]. BCX4430 is active in vitro against the Ebola virus and did not display any significant mutagenicity [73]. The clinical benefit of this small molecule drug against Zika virus needs to be tested in primates before undergoing clinical trials. 


\section{Antibody-based therapeutics}

Antibodies derived from the blood of Zika-infected people may have the potential to protect developing fetuses from the damages inflicted by the virus. Lab tests showed that one of the monoclonal antibodies, ZIKV-117, efficiently neutralized five different Zika strains, which represents the worldwide diversity of the virus [75]. ZIKV-117 was injected into dozens of pregnant mice either one day before or one day after they had been infected with Zika. The antibody treatment markedly reduced the amount of Zika virus in the blood and brain tissues of the pregnant mice, and also reduced the levels of virus found in the placenta and the brain of the unborn fetus [75]. The placenta was not damaged and the fetuses looked normal relative to uninfected animals. In most cases, the antibodies were 95 to 100 percent protective of the fetus [75].

ZIKV-117 works by preventing the reorganization of $\mathrm{E}$ protein monomers into fusogenic trimers in the acidic environment of endosomes [76]. The investigators of this seminal study hope to proceed to human clinical trials for the antibody therapy. They are currently making plans to test the antibody treatment in monkeys.

Two other monoclonal antibodies showed postexposure protection in mice against Zika virus infection [77]. Isolation of 13 specific human monoclonal antibodies from a single patient infected with ZIKV has been reported. Two of the isolated antibodies (Z23 and Z3L1) demonstrated potent ZIKV-specific neutralization in vitro without binding or neutralizing activity against strains 1 to 4 of DENV, the closest relative to ZIKV [77]. Structural studies revealed that Z23 and Z3L1 bound to tertiary epitopes in envelope protein domain I, II, or III, indicating potential targets for ZIKV-specific therapy [77].

\section{Combinational therapy}

Combination treatments using one compound from each category (neuroprotective and antiviral) have shown increased protection of human neural progenitors and astrocytes from ZIKV-induced cell death [41]. Reports have demonstrated that various anti-influenza compounds show some degree of anti-Zika activity [64]. We speculate that combinational treatments with monoclonal neutralizing antibody like ZIKV-117, Z23 or Z3L1 and an antiviral drug, such as an antiinfluenza drug, may result in better treatment outcomes in Zika infection. Early in the Ebola epidemic, several patients received a combination of ZMapp antibody and the anti-viral Favipiravir, which led to recovery $[78,79]$. Reports of enhanced production of proinflammatory cytokines/ chemokines $[57,58,80]$, which are associated with Zika pathogenicity, suggest that anti-inflammatory treatments could ameliorate disease outcomes. We hypothesize that a good candidate for combinational treatment would be one of the immunomodulatory therapeutics, such as AAL-R, that has undergone FDA approval. It has been shown that dampening the host's inflammatory response against influenza virus using an immunomodulatory agent, AAL-R given in combination with anti-influenza drug oseltamivir, provides significantly higher protection (96\%) from mortality in mice infected with pathogenic influenza virus over that of drug oseltamivir alone (50\%) [81]. It is possible that coadministration of one of the immunomodulatory agents discussed above and an anti-HCV drug, like ribavirin (NS5A inhibitor) and grazoprevir (NS3/4A protease inhibitor), or an anti-influenza drug like oseltamivir would provide better treatment outcomes in Zika infection.

\section{Antibiotics and their anti-ZIKV activity}

Recently three antibiotics have demonstrated the ability to reduce ZIKV proliferation in cell culture systems. Duramycin, a cyclic peptide, was shown to block ZIKV infection of several primary placental cell types from mid- and late gestation [82]. It has been suggested that duramycin inhibits the binding of ZIKV to TIM1, a protein that is universally expressed across the uterine interface during gestation. Daptomycin is a lipopeptide antibiotic that inserts into cell membranes rich in Phosphatidylglycerol (PG), and impacts PG-rich late endosomal membranes, which are critical for viral entry. Daptomycin showed variable effectiveness in reducing ZIKV replication in various human cell types $[83,84]$. Azithromycin, a macrolide antibiotic, has been shown to prevent ZIKV from infecting fetal brain tissue cells in vitro [84]. This antibiotic rescued ZIKV-induced cytopathic effects with low toxicity in primary screens and is generally regarded as safe for use in pregnancy [85]. Of note, duramycin is not recommended by the FDA for use during pregnancy. No harm has been found with the use of daptomycin in animals during pregnancy; however, adequate studies have not been conducted in pregnant women.

The mechanisms of action for these antibiotics are largely unknown. Further studies are required to determine whether these antibiotics, other inhibitors, or any combination thereof are capable of inhibiting ZIKV infection in vivo.

\section{Translational Challenges and Prospects}

There is an urgent need to identify therapies that are effective and safe, and well-designed clinical trials are the fastest and most reliable way to achieve that goal. All the candidate drugs against ZIKV need to first to pass through screening assays or animal experiments before being utilized in human trials. However, ZIKV models, such as those in rodents and non-human primates, do not completely recapitulate the immunological aspects and pathogenesis of ZIKV infection as it occurs in humans. As a consequence, there may be considerable differences in treatment efficacy across different animal models of ZIKV infection.

ZIKV poses many unique challenges. For example, drugs and vaccines will be targeted towards immunocompromised populations (pregnant mothers and fetuses), which risks stimulating harmful immune responses (either autoimmune or antibody-dependent enhancement of infection in those with previous flavivirus exposure). Furthermore, the mildly symptomatic infection may result in delayed treatments and increased risk of transmission to others. Having considered these circumstances and the multiple routes of transmission (arthropod vector, sexual, blood-borne, and other body fluids), we postulate that frequent travellers to ZIKV endemic areas, and those residing in endemic areas with suspected exposures could be target populations for drug testing.

The diminishing number of Zika patients may hamper the human trials of anti-Zika drugs. Renewing efforts have to be made to scale up the production of promising drugs. Only those therapies available in large enough quantities to treat the thousands in need of immediate treatment will be useful in an epidemic situation. Some of the investigational drugs require refrigeration and have to be administered by injections, which is less ideal with regard to patient compliance in outbreak scenarios. These concerns underline the importance for adopting better treatment strategies. Future tests will need to better reflect the real-world situation of most Zika-infected people. The 12day asymptomatic period post-ZIKV infection and viral tropism to developing neural cells in the fetus reinforces that therapeutic strategies initiated earlier are more likely to slow down the disease.

In the absence of an appropriate animal model for carrying out investigational research, development of a cell-based assay that 
measures protection from the ZIKV-induced cytopathic effects could serve as a high-throughput screening assay for discovering novel antiZIKV inhibitors. Concerted efforts are needed to establish new animal models that can mimic signs of neurological disease, thus providing a platform for the development of potential therapeutics and vaccines. The cost of therapy needs to be within the reach of populations affected by Zika infection in endemic areas. There is an urgent need for a screening method and strategy that is focused on highlighting potential anti-ZIKV compounds that can be further advanced via rigorous validation and optimization.

\section{Conclusion}

Zika virus infection in humans has the potential to burgeon into a full-blown epidemic as a result of factors like population expansion, urbanization, globalization and climate change. Due to the history of multiple outbreaks of Zika virus and the likelihood of future exposure, the need for developing efficacious pre- and post-exposure treatments is of paramount importance. The CDC has emphasized that while much progress has been made, the fight against Zika is not over.

In the absence of FDA-approved therapeutics, we have highlighted the justification of repurposing existing drugs to confront the threats posed by Zika. The importance of further assessing the therapeutic opportunities in drug combinations needs to be considered, as the use of combinational therapy is likely to extend the treatment window and improve efficacy.

Animal and human studies indicate that Zika infection triggered an excessive inflammatory response in the nervous system. We postulate that treatment of Zika patients with immunomodulatory agents already approved by the FDA would provide a beneficial anti-inflammatory response and bring clinical benefits to Zika patients in post-exposure conditions.

Research on different aspects of the viral life cycle remains a priority in developing antivirals. Animal studies have demonstrated that miRNAs are involved in viral disease and controlling inflammation. Novel anti-Zika drug development approaches may include miRNAtargeting, which could be an effective strategy for selective control of tissue-tropism and pathogenicity.

Small molecule-based, oral treatment of ZIKV infections is of particular appeal in remote outbreak settings, because of reduced logistical challenges compared to intravenous administration. Comparison of antiviral activity data, safety, cost, and accessibility will validate the efficacy of candidate compounds in treating Zika patients. Preventive measures such as mosquito vector abatement and vaccine development, in conjunction with the identification and development of inhibitors with therapeutic potential are essential endeavors in confronting this emerging threat.

\section{Acknowledgements}

Authors report no conflicts of interest. All authors have contributed extensively to the work presented in this paper. $\mathrm{AH}$ has conceived the concept and design of the paper. ABP has participated in the revision process, and created figures and tables for the present manuscript.

\section{References}

1. Dick GW (1952) Zika virus (ii) pathogenicity and physical properties. Trans R Soc Trop Med Hyg 46: 521-534.

2. Dick GW, Haddow AJ (1952) Uganda S virus; a hitherto unrecorded virus isolated from mosquitoes in Uganda. I. Isolation and pathogenicity. Trans R Soc Trop Med Hyg 46: 600-618.

3. Macnamara FN (1954) Zika virus: a report on three cases of human infection during an epidemic of jaundice in Nigeria. Trans R Soc Trop Med Hyg 48 139-145

4. Duffy MR, Chen TH, Hancock WT, Powers AM, Kool JL, et al (2009) Zika virus outbreak on Yap Island, Federated States of Micronesia. N Engl J Med 360 2536-2543

5. Mlakar J, Korva M, Tul N, PopoviÄ M, Poljšak-Prijatelj M, et al. (2016) Zika Virus Associated with Microcephaly. N Engl J Med 374: 951-958.

6. https://www.cdc.gov/zika/

7. Knudsen $A B$ (1995) Global distribution and continuing spread of Aedes albopictus. Parassitologia 37: 91-97.

8. Grard G, Caron M, Mombo IM, Nkoghe D, Mboui Ondo S, et al. (2014) Zika virus in Gabon (Central Africa)-2007: a new threat from Aedes albopictus? PLoS Negl Trop Dis 8: e2681.

9. Diagne CT, Diallo D, Faye O, Ba Y, Faye O, et al. (2015) Potential of selected Senegalese Aedes spp. mosquitoes (Diptera: Culicidae) to transmit Zika virus. BMC Infect Dis 15: 492.

10. Foy BD, Kobylinski KC, Chilson Foy JL, Blitvich BJ, Travassos da Rosa A, et al. (2011) Probable non-vector-borne transmission of Zika virus, Colorado, USA Emerg Infect Dis 17: 880-882.

11. Musso D, Nhan T, Robin E, Roche C, Bierlaire D, et al. (2014) Potential for Zika virus transmission through blood transfusion demonstrated during an outbreak in French Polynesia, November 2013 to February 2014. Euro Surveil 19: 20761.

12. Mansuy JM, Dutertre M, Mengelle C, Fourcade C, Marchou B, et al. (2016) Zika virus: high infectious viral load in semen, a new sexually transmitted pathogen? Lancet Infect Dis 16: 405.

13. Bonaldo MC, Ribeiro IP, Lima NS, Dos Santos AA, Menezes LS, et al. (2016) Isolation of Infective Zika Virus from Urine and Saliva of Patients in Brazil. PLoS Negl Trop Dis 10: e0004816.

14. Dupont-Rouzeyrol M, Biron A, O'Connor O, Huguon E, Descloux E (2016) Infectious Zika viral particles in breastmilk. Lancet 387: 1051.

15. Paz-Bailey G, Rosenberg ES, Doyle K, Munoz-Jordan J, Santiago GA, et al. (2017) Persistence of Zika Virus in Body Fluids-Preliminary Report. N Engl J Med.

16. http://www.mlive.com/weather/index.ssf/2017/05/early_signs_a_new_el_nino_ may_1.html

17. Cordeiro MT, Pena LJ, Brito CA, Gil LH, Marques ET (2016) Positive IgM for Zika virus in the cerebrospinal fluid of 30 neonates with microcephaly in Brazil. Lancet 387: 1811-1812.

18. Schuler-Faccini L, Ribeiro EM, Feitosa IM, Horovitz DD, Cavalcanti DP, et al. (2016) Possible association between Zika virus infection and microcephalyBrazil, 2015. MMWR Morb Mortal Wkly Rep 65: 59-62.

19. Nowakowski TJ, Pollen AA, Di Lullo E, Sandoval-Espinosa C, Bershteyn M, et al. (2016) Expression analysis highlights AXL as a candidate Zika virus entry receptor in neural stem cells. Cell Stem Cell 18: 591-596.

20. Hamel R, Dejarnac O, Wichit S, Ekchariyawat P, Neyret A, et al. (2015) Biology of Zika virus infection in human skin cells. J Virol 89: 8880-8896.

21. http://apps.who.int/iris/bitstream/10665/204475/1/WHO_ZIKV_MOC_16.3_ eng.pdf?ua $=1$

22. Calvet G, Aguiar RS, Melo AS, Sampaio SA, de Filippis I, et al. (2016) Detection and sequencing of Zika virus from amniotic fluid of fetuses with microcephaly in Brazil: a case study. Lancet Infect Dis 16: 653-660.

23. Besnard M, Lastere S, Teissier A, Cao-Lormeau V, Musso D (2014) Evidence of perinatal transmission of Zika virus, French Polynesia, December 2013 and February 2014. Euro Surveil 19: 20751.

24. Musso D, Roche C, Robin E, Nhan T, Teissier A, et al. (2015) Potential sexual transmission of Zika virus. Emerg Infect Dis 21: 359-361.

25. Brasil P, Nielsen-Saines K (2016) More pieces to the microcephaly-Zika virus puzzle in Brazil. Lancet Infect Dis 16: 1307-1309.

26. Carteaux G, Maquart M, Bedet A, Contou D, Brugieres P, et al. (2016) Zika virus associated with Meningoencephalitis. N Engl J Med 374: 1595-1596

27. Mecharles S, Herrmann C, Poullain P, Tran TH, Deschamps N, et al. (2016) Acute myelitis due to Zika virus infection. Lancet 387: 1481 
Citation: Haque A, Pant AB (2017) Potential Therapeutics: To Hold in the Fight against Zika Virus. J Bioanal Biomed 9: 177-185. doi:10.4172/1948593X.1000175

28. Lucchese G, Kanduc D (2016) Zika virus and autoimmunity: From microcephaly to Guillain-Barre syndrome, and beyond. Autoimmun Rev 15: 801-808.

29. Meertens L, Labeau A, Dejarnac O, Cipriani S, Sinigaglia L, et al. (2017) Ax mediates ZIKA virus entry in human glial cells and modulates innate immune responses. Cell Rep 18: 324-333.

30. Richard AS, Shim BS, Kwon YC, Zhang R (2017) AXL-dependent infection of human fetal endothelial cells distinguishes Zika virus from other pathogenic flaviviruses. Proc Natl Acad Sci U S A 114: 2024-2029.

31. Bhattacharyya S, Zagorska A, Lew ED, Shrestha B, Rothlin CV, et al. (2013) Enveloped viruses disable innate immune responses in dendritic cells by direct activation of TAM receptors. Cell Host Microbe 14: 136-147.

32. Meertens L, Carnec X, Lecoin MP, Ramdasi R, Guivel-Benhassine F, et al. (2012) The TIM and TAM families of phosphatidylserine receptors mediate dengue virus entry. Cell Host Microbe 12: 544-557.

33. Bowen JR, Quicke KM, Maddur MS, O'Neal JT, McDonald CE, et al. (2017) Zika virus antagonizes type i interferon responses during infection of human dendritic cells. PLoS Pathog 13: e1006164.

34. Grant A, Ponia SS, Tripathi S, Balasubramaniam V, Miorin L, et al. (2016) Zika virus targets human STAT2 to inhibit type i interferon signaling. Cell Host Microbe 19: 882-890.

35. Haque A, Hober D, Blondiaux J (2015) Addressing therapeutic options for Ebola virus infection in current and future outbreaks. Antimicrob Agents Chemother 59: $5892-5902$

36. Perera DR, Western KA, Schultz MG (1970) Niclosamide treatment of cestodiasis. Clinicial trials in the United States. Am J Trop Med Hyg 19: 610-612.

37. Ditzel J, Schwartz M (1967) Worm cure without tears. The effect of niclosamide on taeniasis saginata in man. Acta Med Scand 182: 663-664.

38. Wang YM, Lu JW, Lin CC, Chin YF, Wu TY, et al. (2016) Antiviral activities of niclosamide and nitazoxanide against chikungunya virus entry and transmission. Antiviral Res 135: 81-90.

39. Fang J, Sun L, Peng G, Xu J, Zhou R, et al. (2013) Identification of three antiviral inhibitors against Japanese encephalitis virus from library of pharmacologically active compounds 1280. PLoS ONE 8: e78425.

40. Jurgeit A, McDowell R, Moese S, Meldrum E, Schwendener R, et al. (2012) Niclosamide is a proton carrier and targets acidic endosomes with broad antiviral effects. PLoS Pathog 8: e1002976.

41. Xu M, Lee EM, Wen Z, Cheng Y, Huang WK, et al. (2016) Identification of small-molecule inhibitors of Zika virus infection and induced neural cell death via a drug repurposing screen. Nat Med 22: 1101-1107.

42. Khanim FL, Merrick BA, Giles HV, Jankute M, Jackson JB, et al. (2011) Redeployment-based drug screening identifies the anti-helminthic niclosamide as anti-myeloma therapy that also reduces free light chain production. Blood Cancer J 1: e39.

43. World Health Organization (WHO) (1995) WHO model prescribing information: drugs used in parasitic diseases ( $2^{\text {nd }}$ edn.). Geneva.

44. Tang H, Hammack C, Ogden SC, Wen Z, Qian X, et al. (2016) Zika virus infects human cortical neural progenitors and attenuates their growth. Cell Stem Cell 18: $587-590$

45. Iorio F, Bosotti R, Scacheri E, Belcastro V, Mithbaokar P, et al. (2010) Discovery of drug mode of action and drug repositioning from transcriptional responses. Proc Natl Acad Sci U S A 107: 14621-14626.

46. Savarino A, Boelaert JR, Cassone A, Majori G, Cauda R (2003) Effects of chloroquine on viral infections: an old drug against today's diseases? Lancet Infect Dis 3: 722-727.

47. Browning DJ, Lee C (2014) Relative sensitivity and specificity of 10-2 visual fields, multifocal electroretinography, and spectral domain optical coherence tomography in detecting hydroxychloroquine and chloroquine retinopathy. Clin Ophthalmol 8: 1389-1399.

48. Wolfe MS, Cordero JF (1985) Safety of chloroquine in chemosuppression of malaria during pregnancy. Br Med J 290: 1466-1467.

49. Levy M, Buskila D, Gladman DD, Urowitz MB, Koren G (1991) Pregnancy outcome following first trimester exposure to chloroquine. Am J Perinatol 8: 174-178.
50. Olsen NJ, Schleich MA, Karp DR (2013) Multifaceted effects of hydroxychloroquine in human disease. Semin Arthritis Rheum 43: 264-272.

51. Smit JM, Moesker B, Rodenhuis-Zybert I, Wilschut J (2011) Flavivirus cell entry and membrane fusion. Viruses 3: 160-171

52. Delvecchio R, Higa LM, Pezzuto $P$, Valadão AL, Garcez PP, et al. (2016) Chloroquine, an endocytosis blocking agent, inhibits zika virus infection in different cell models. Viruses 8: 322.

53. Kappos L, Antel J, Comi G, Montalban X, O'Connor P, et al. (2006) Ora fingolimod (FTY720) for relapsing multiple sclerosis. N Engl J Med 355: 1124 1140 .

54. Brinkmann V (2009) FTY720 (fingolimod) in Multiple Sclerosis: therapeutic effects in the immune and the central nervous system. Br J Pharmacol 158 1173-1182.

55. Yanagawa Y, Sugahara K, Kataoka H, Kawaguchi T, Masubuchi Y, et al. (1998) FTY720, a novel immunosuppressant, induces sequestration of circulating mature lymphocytes by acceleration of lymphocyte homing in rats. II. FTY720 prolongs skin allograft survival by decreasing $T$ cell infiltration into grafts but not cytokine production in vivo. J Immunol 160: 5493-5499.

56. Brinkmann V, Pinschewer DD, Feng L, Chen S (2001) FTY720: altered lymphocyte traffic results in allograft protection. Transplantation 72: 764-769.

57. Ornelas AM, Pezzuto P, Silveira PP, Melo FO, Ferreira TA, et al. (2017) Immune activation in amniotic fluid from Zika virus-associated microcephaly. Ann Neurol 81: 152-156.

58. Galliez RM, Spitz M, Rafful PP, Cagy M, Escosteguy C, et al. (2016) Zika virus causing encephalomyelitis associated with immunoactivation. Open Forum Infect Dis 3: ofw203.

59. Walsh KB, Teijaro JR, Wilker PR, Jatzek A, Fremgen DM, et al. (2011) Suppression of cytokine storm with a sphingosine analog provides protection against pathogenic influenza virus. Proc Natl Acad Sci USA 108: 12018-12023.

60. Li G, Poulsen M, Fenyvuesvolgyi C, Yashiroda Y, Yoshida M, et al. (2017) Characterization of cytopathic factors through genome-wide analysis of the Zika viral proteins in fission yeast. Proc Natl Acad Sci USA 114: E376-E385.

61. Laplante M, Sabatini DM (2012) mTOR signaling in growth control and disease. Cell 149: 274-293.

62. Loewith R, Hall MN (2011) Target of rapamycin (TOR) in nutrient signaling and growth control. Genetics 189: 1177-1201.

63. Mannick JB, Del Giudice G, Lattanzi M, Valiante NM, Praestgaard J, et al (2014) mTOR inhibition improves immune function in the elderly. Sci Trans Med 6: 268ra179.

64. Kuivanen S, Bespalov MM, Nandania J, lanevski A, Velagapudi V, et al. (2017) Obatoclax, saliphenylhalamide and gemcitabine inhibit Zika virus infection in vitro and differentially affect cellular signaling, transcription and metabolism. Antiviral Res 139: 117-128.

65. Kwo PY, Lawitz EJ, McCone J, Schiff ER, Vierling JM, et al. (2010) Efficacy of boceprevir, an NS3 protease inhibitor, in combination with peginterferon alfa-2b and ribavirin in treatment-naive patients with genotype 1 hepatitis $C$ infection (SPRINT-1): an open-label, randomised, multicentre phase 2 trial. Lancet 376 : 705-716.

66. Jacobson IM, McHutchison JG, Dusheiko G, Di Bisceglie AM, Reddy KR, et al. (2011) Telaprevir for previously untreated chronic hepatitis $C$ virus infection. N Engl J Med 364: 2405-2416.

67. Bacon BR, Gordon SC, Lawitz E, Marcellin P, Vierling JM, et al. (2011) Boceprevir for previously treated chronic HCV genotype 1 infection. N Engl J Med 364: 1207-1217.

68. Malcolm BA, Liu R, Lahser F, Agrawal S, Belanger B, et al. (2006) SCH 503034 a mechanism-based inhibitor of hepatitis C virus NS3 protease, suppresses polyprotein maturation and enhances the antiviral activity of alpha interferon in replicon cells. Antimicrob Agents Chemother 50: 1013-1020.

69. Lain S, Riechmann JL, Martin MT, Garcia JA (1989) Homologous potyvirus and flavivirus proteins belonging to a superfamily of helicase-like proteins. Gene 82: 357-362.

70. Lescar J, Luo D, Xu T, Sampath A, Lim SP, et al. (2008) Towards the design of antiviral inhibitors against flaviviruses: the case for the multifunctional NS3 protein from Dengue virus as a target. Antiviral Res 80: 94-101. 
Citation: Haque A, Pant AB (2017) Potential Therapeutics: To Hold in the Fight against Zika Virus. J Bioanal Biomed 9: 177-185. doi:10.4172/1948593X.1000175

71. Bullard-Feibelman KM, Govero J, Zhu Z, Salazar V, Veselinovic M, et al. (2017) The FDA-approved drug sofosbuvir inhibits Zika virus infection. Antiviral Res 137: $134-140$

72. Tian H, Ji X, Yang X, Xie W, Yang K, et al. (2016) The crystal structure of Zika virus helicase: basis for antiviral drug design. Protein Cell 7: 450-454.

73. Warren TK, Wells J, Panchal RG, Stuthman KS, Garza NL, et al. (2014) Protection against filovirus diseases by a novel broad-spectrum nucleoside analogue BCX4430. Nature 508: 402-405.

74. Julander JG, Siddharthan V, Evans J, Taylor R, Tolbert K, et al. (2017) Efficacy of the broad-spectrum antiviral compound BCX4430 against Zika virus in cell culture and in a mouse model. Antiviral Res 137: 14-22.

75. Sapparapu G, Fernandez E, Kose N, Bin C, Fox JM, et al. (2016) Neutralizing human antibodies prevent Zika virus replication and fetal disease in mice. Nature 540: 443-447.

76. Hasan SS, Miller A, Sapparapu G, Fernandez E, Klose T, et al. (2017) A human antibody against Zika virus crosslinks the $E$ protein to prevent infection. Nat Commun 8: 14722

77. Wang Q, Yang H, Liu X, Dai L, Ma T, et al. (2016) Molecular determinants of human neutralizing antibodies isolated from a patient infected with Zika virus. Sci Transl Med 8: 369ra179.

78. Qiu X, Wong G, Fernando L, Audet J, Bello A, et al. (2013) mAbs and Advectored IFN- $\alpha$ therapy rescue Ebola-infected nonhuman primates when administered after the detection of viremia and symptoms. Sci Transl Med 5 : 207 ra143.

79. Qiu X, Wong G, Fernando L, Ennis J, Turner JD, et al. (2013) Monoclona antibodies combined with adenovirus-vectored interferon significantly extend the treatment window in Ebola virus-infected guinea pigs. J Virol 87: 77547757.

80. Tappe D, Perez-Giron JV, Zammarchi L, Rissland J, Ferreira DF, et al. (2016) Cytokine kinetics of Zika virus-infected patients from acute to reconvalescent phase. Med Microbiol Immunol 205: 269-273.

81. Oldstone MB, Teijaro JR, Walsh KB, Rosen H (2013) Dissecting influenza virus pathogenesis uncovers a novel chemical approach to combat the infection. Virology 435: 92-101.

82. Tabata T, Petitt M, Puerta-Guardo H, Michlmayr D, Wang C, et al. (2016) Zika virus targets different primary human placental cells, suggesting two routes for vertical transmission. Cell Host Microbe 20: 155-166.

83. Barrows NJ, Campos RK, Powell ST, Prasanth KR, Schott-Lerner G, et al. (2016) A screen of fda-approved drugs for inhibitors of Zika virus infection. Cell Host Microbe 20: 259-270.

84. Retallack H, Di Lullo E, Arias C, Knopp KA, Laurie MT, et al. (2016) Zika virus cell tropism in the developing human brain and inhibition by azithromycin. Proc Natl Acad Sci USA 113: 14408-14413.

85. Lin KJ, Mitchell AA, Yau WP, Louik C, Hernández-Díaz S (2013) Safety of macrolides during pregnancy. Am J Obstet Gynecol 208: 221 